Iwona Lupa-Wójcik

Pedagogical University of Krakow

\title{
STUDENT ATTITUDES TOWARD THE USE OF CHATBOTS IN CUSTOMER SERVICE
}

The article addresses an important and current problem related to the use of chatbots in customer service, according to students. The aim of the research was to recognize the attitude of respondents to chatbots in the context of customer service and to determine the development prospects for their use in this area. The research examined the recognizability of chatbots among respondents, the frequency of contact between respondents and chatbots, as well as the impression (feeling) of respondents using chatbots. As a research method, student survey was used. Research has shown that chatbots are still a new tool for respondents, however they have great potential in customer service, as they are user-friendly and are very useful in the area of customer service. Research has shown that the role of chatbots in this area should increase. This can have a good effect on the image of the brand / company.

Key words: chatbots, customer service, social media, marketing automation, helpline, Messenger JEL Code: M3

\section{Introduction}

Currently, in the age of social media, the need for instant customer service has become a pressing problem for companies. They are required to respond immediately to thousands of customer inquiries that appear very quickly. It is not surprising that the marketing automation trend is developing strongly, and the use of chatbots in customer service as well.

The purpose of this article is to recognize the attitude of respondents to chatbots in the context of customer service and to determine the prospects for their use in this regard from the point of view of users. As the main hypothesis it was assumed that the vast majority of respondents had contact with chatbots, although in many cases they were not aware of it, and assess their usefulness in the field of customer service. The role of chatbots in customer service should increase and it can have a good impact on the image of the brand / company.

\section{Literature review}

Over the last few years, many scientific studies have been conducted on chatbots and their use in customer service. In 2007, Bayan Abu Shawar and Eric Atwell discussed their usefulness in various areas of life, such as education, information retrieval, business or e-commerce". The authors defined them as "computer programs that interact with users using natural languages"2 and they pointed out that the beginnings of this type of technology date back to the 1960 's. Also, in 2007, works by Huang, Jizhou, Ming Zhou,

${ }^{1}$ B.A. Shawar, E. Atwell: Chatbots: are they really useful?,Ldv forum. Vol. 22. No. 1. 2007.

${ }^{2}$ Ibidem, p. 29. 
and Dan Yang, similarly defined chatbot as a "conversational agent that interacts with users in a certain domain or on a certain topic with natural language sentences" ${ }^{\prime 3}$. R. Dale in 2016 wrote a work entitled "The return of the chatbots", exploring what had changed in the last decades due to the use of chatbots, since they have such a long history ${ }^{4}$. During this time, many studies were carried out related to the technical aspects of using chatbots in conversation with people. For example, authors have wondered why people use chatbots ${ }^{5}$. An interesting study is also shown by J. Hill, W.R. Ford and I.G. Farreras, presenting "a comparison between human-human online conversations and humanchatbot conversations" $"$. This type of research has undoubtedly contributed to the development of chatbots in customer service.

In the area of communication with clients, chatbots have also been used by companies for a long time. Understanding the concept of chatbot very widely (as in this article), one can give many examples of using chatbots in customer service, e.g. automatic e-mails sent after making a purchase in an online store. Courier companies also use chatbots for customer service on a large scale. One can read a lot about chatbots in customer communication in A. Braun's book from $2003^{7}$. However, the real revolution associated with the use of chatbots in customer service came with the development of social media in this area, which was noticed, for example, by A. Xu, Z. Liu, Y. Guo, V. Sinha, \& R. Akkiraj ${ }^{8}$.

Currently, chatbots are present on many company websites, providing customer support while making purchase decisions and even replacing the helpline to some extent. Many companies develop chatbots on Messenger, which is currently the most popular communicator in Poland and in the world ${ }^{9}$, closely related to Facebook. It is anticipated that by $2020,80 \%$ of companies in the world will use chatbots, and by 2022, banks may automate up to $90 \%$ of customer interaction with chatbots ${ }^{10}$. However chatbots on Messenger are still new to users, and their wider use in customer service may raise doubts. For example " $46 \%$ of Internet users in the US would rather receive online support from a live person, even if the chatbot can save them a lot of time" ${ }^{\text {"11 }}$. Customers fear that chatbots hinder contact with a live person (50.7\%) and give too many unhelpful answers $(47.5 \%)^{12}$. Therefore the use of chatbots in customer service is a big challenge for companies.

${ }^{3}$ J. Huang, M. Zhou, D. Yang: Extracting Chatbot Knowledge from Online Discussion Forums, IJCAI. Vol. 7. 2007, p. 423.

${ }^{4}$ R. Dale: The return of the chatbots, Natural Language Engineering 22.5 (2016): 811-817.

${ }^{5}$ P.B. Brandtzaeg, A. Følstad: Why people use chatbots, International Conference on Internet Science. Springer, Cham, 2017

${ }^{6}$ J. Hill, W.R. Ford, I.G. Farreras: Real conversations with artificial intelligence: A comparison between human-human online conversations and human-chatbot conversations." Computers in Human Behaviour 49 (2015): 245-250

${ }^{7}$ A. Braun, Chatbots in der Kundenkommunikation. Springer-Verlag, 2013.

${ }^{8} \mathrm{Xu}$, Anbang, et al.: A new chatbot for customer service on social media. Proceedings of the $2017 \mathrm{CHI}$ Conference on Human Factors in Computing Systems. ACM, 2017.

${ }^{9} \mathrm{https}: / /$ napoleoncat.com/stats/ (28.02.2019).

${ }^{10}$ Chatbot Report 2019: Global Trends and Analysis, https://chatbotsmagazine.com/chatbot-report-2019-globaltrends-and-analysis-a $487 \mathrm{afec} 05 \mathrm{~b}(14.10 .2019)$.

${ }^{11}$ Ibid.

${ }^{12}$ Ibid. 


\section{Methodology}

The aim of the research was to recognize the attitude of respondents to chatbots in the context of customer service and to determine the development prospects for their use in this area. The following sub-objectives have been indicated:

- determining the recognizability of chatbots among respondents,

- recognition of the frequency of contact of respondents with chatbots,

- examining the feelings of respondents in relation to chatbots,

- determining the suitability of chatbots in customer service,

- recognizing the possibilities of developing the use of chatbots in the context of customer service.

As the main hypothesis, it was assumed that the vast majority of respondents had contact with chatbots, although in many cases they were not aware of it. They assess relatively well the usefulness of chatbots in the field of customer service. The role of chatbots in customer service should increase and it can have a good impact on the image of the brand / company.

The following specific hypotheses were formulated:

H1: Respondents, despite the very frequent use of various types of internet services (eg e-mail, Facebook, Messenger, internet shopping), in many cases cannot determine if they have ever met with any chatbot.

$\mathrm{H} 2$ : Respondents can determine contact with a given chatbot only after being asked about it directly (giving an example).

H3: Respondents positively assess the usefulness of pop-ups chatbots on the websites of companies and of chatbots in Messenger.

H4: In the opinion of the respondents, chatbots have a beneficial effect on the image of the brand or company that uses them in customer service.

H5: According to respondents, chatbots are a good alternative to other communication channels in the field of customer service, and their development in this direction is desirable.

A survey was used to verify the hypotheses. The questionnaire consisted of 12 questions regarding the attitude of respondents to chatbots, as well as the usefulness of these tools in customer service. In addition, the questionnaire contained two metrics questions about gender and age of the respondents. The questionnaire was sent in electronic form to students of the Pedagogical University of Krakow (Poland). Students constitute a group of "technologically advanced" consumers, which means that they set the directions of trends. All surveyed students are internet users. If the hypothesis that the knowledge and use of chatbots in this group is small is confirmed, it should be assumed that also in other groups of Internet users these attitudes will not be different.

The research sample was 120 people. The study is a pilot. Respondents were mainly women $(83 \%)$. Men constituted the decided minority $(17 \%)$. The respondents were mainly aged 18-25 (96\%). The research was carried out in February 2019.

\section{Findings}

To verify the hypotheses, information on how often the respondents use selected internet services, such as e-mail, Facebook, Messenger or an online store (including Allegro - the 
Polish equivalent of eBay), was determined. Using these services means very high probability, or rather the certainty that the respondents had contact with chatbots. For example, the use of courier services was also been verified, as they use chatbots on a large scale in customer service (e.g. for tracking shipments). The information collected on this topic is summarized in Figure 1.

As many as $95 \%$ of respondents very often use Messenger (only $2 \%$ indicated that they never do), and nearly $80 \%$ - Facebook. In addition, $42 \%$ of respondents sometimes shop online, and $33 \%$ - rather often. Many people also use, for example, courier services (36\% - sometimes, $31 \%$ - rather often).

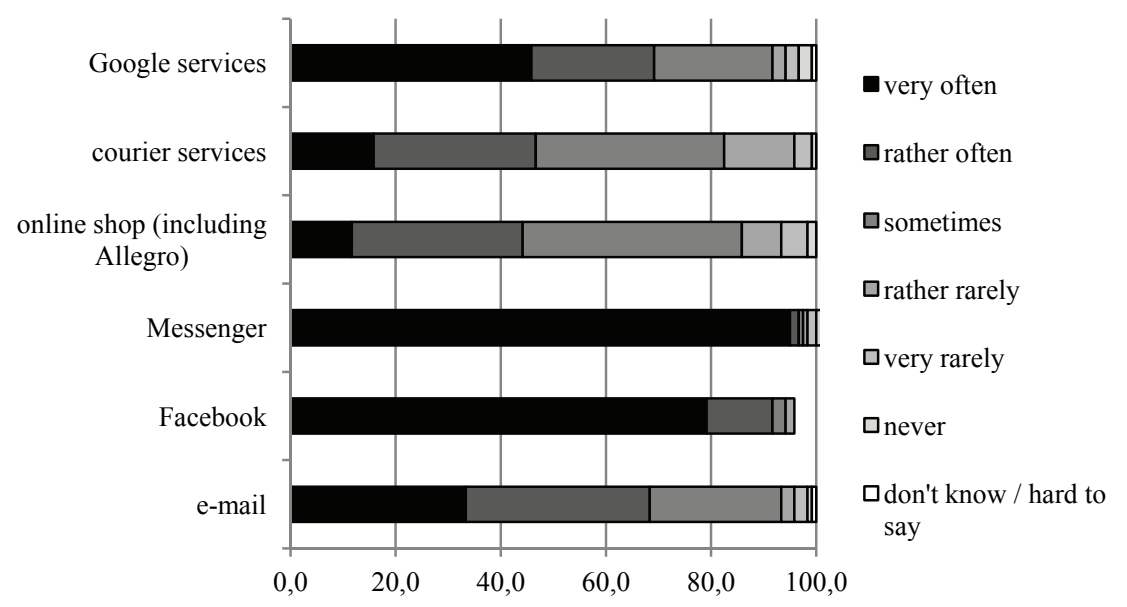

Figure 1.The frequency of use of selected services by respodents $(\mathrm{N}=120)$ Source: Author's own research.

Generally, surveyed students use selected internet services on a large scale. It was examined whether they declared contact with a chatbot (Figure 2).

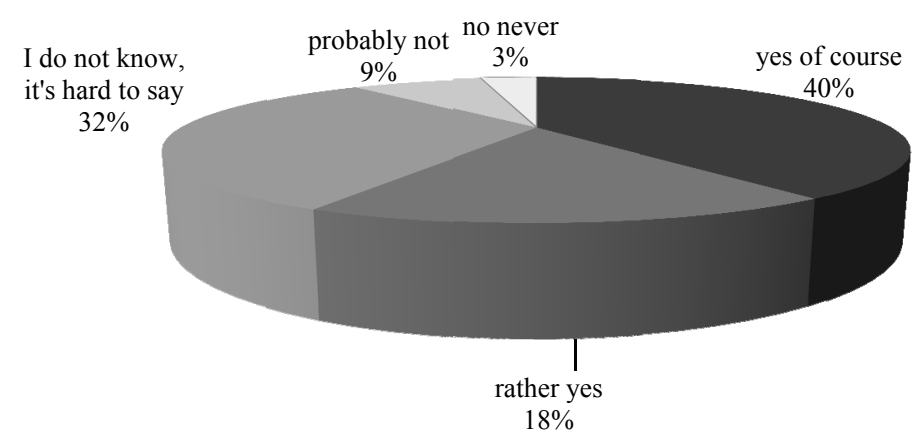

Figure 2. Contact of respondents with chatbot (according to themselves)( $\mathrm{N}=120)$

Source: Author's own research. 
$40 \%$ of the surveyed students decided that they had contact with a chatbot, while $32 \%$ were unable to determine this issue. This data means that there is still an average awareness about chatbots among the respondents. The H1 hypothesis was confirmed that respondents - despite the very frequent use of various types of internet services (e.g. email, Facebook, Messenger, internet shopping) - in many cases cannot determine if they have ever met with any chatbot.

Anticipating such research results, students were asked how often they meet specific chatbots, e.g. pop-up chat windows on websites where they are invited to a conversation about the company's offer etc., Google Assistant, chatbots on Messenger, which provide specific information, e.g. about the weather, about company competitions, etc., chatbots on e-mail (they send information, e.g. about subscribing to a newsletter, about purchasing an item, etc.) or other (Figure 3).

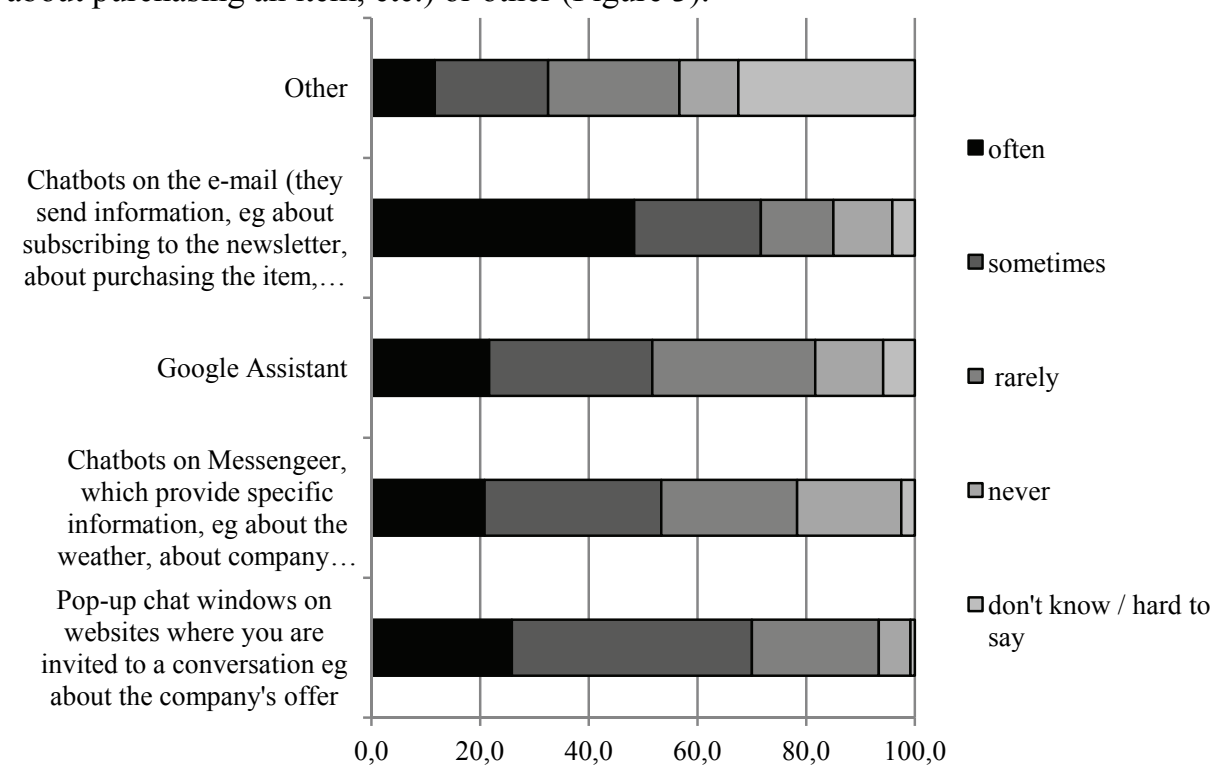

Figure 3. Frequency of contact of respondents with selected chatbots $(\mathrm{N}=120)$ Source: Author's own research.

It turns out that only $6 \%$ of the surveyed students have never encountered chat windows on the websites of various companies, $11 \%$ - with email chat chats and $13 \%$ with Google Assistant. Slightly more of the respondents indicated that they had never met with chatboxes on Messenger. These results confirm the $\mathrm{H} 2$ hypothesis that respondents can determine contact with a given chatbot only after being asked about it directly. In general, however, knowledge and awareness among the studied group of students about chatbots is rather average.

In the further stage of the research, to obtain more precise results, the questions for the respondents were limited to only two types of chatbots: pop-up chat windows on websites and to chatbots on Messenger.

First of all, the ratio of respondents to pop-up chat windows on websites will be discussed. The respondents were asked how often they use this type of chat to get more 
information about, for example, the company's offer (Figure 4). The question contained a screenshot showing an example of such a conversation, so that the respondents understood well what chatbot was being referred to.

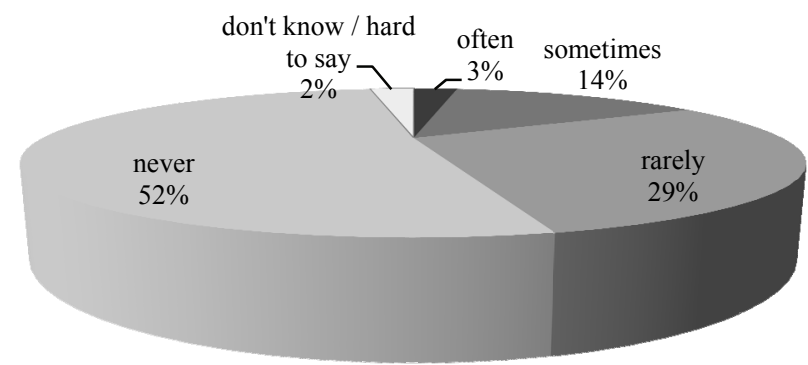

Figure 4. Frequency of use by respondents, chat on the website of a company to obtain more information, e.g. about its offer $(\mathrm{N}=120)$

Source: Author's own research.

The research showed that $52 \%$ of the surveyed students never used chat on a company's website, $29 \%$ use this communication channel rarely, and $14 \%$ - sometimes. Chat on websites is not a popular way of contact between customers and the company, which is not surprising, however - as it turns out - there are a lot of people who use this option.

The respondents who used the chat on the company's website $(\mathrm{N}=55)$ were asked about the extent to which they managed to settle the matter thanks to this form of contact. Answers are presented on Figure 5.

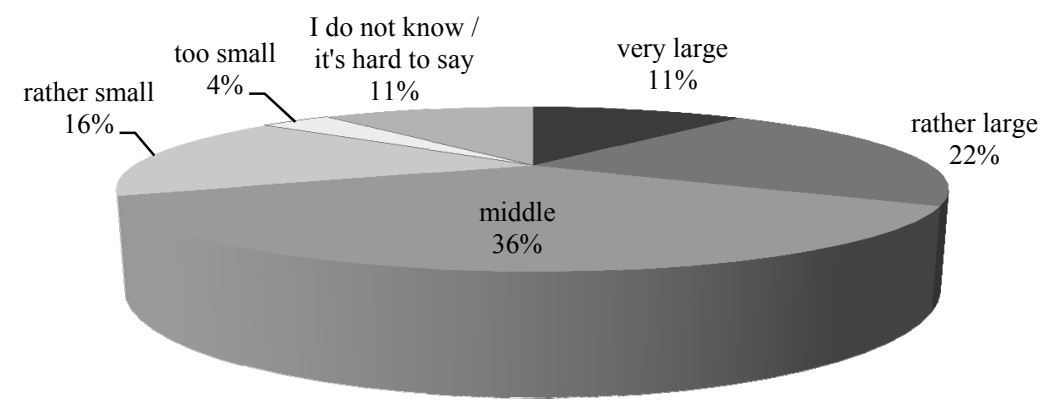

Figure 5. The extent to which respondents managed to settle the matter via chat on the website $(\mathrm{N}=55)$ Source: Author's own research.

Every third respondent stated that $\mathrm{s} / \mathrm{he}$ managed to settle the matter to a medium degree through the channel (36\%), while $22 \%$ - rather large. This would indicate a rather positive assessment of the suitability of this form of customer contact with the company. One can see more on this topic on Figure 6. 


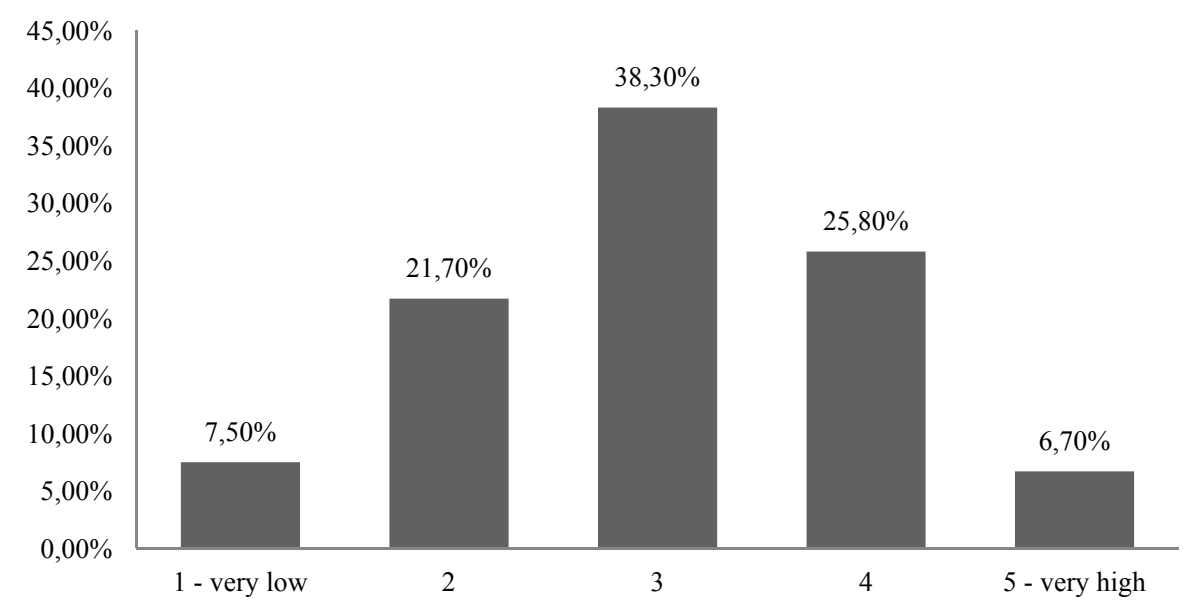

Figure 6. Respondents' assessment of the usefulness of chat on websites for a client seeking information about a company and its offer $(\mathrm{N}=120)$

Source: Author's own research.

Respondents rate the usefulness of popups on websites by an average (38\%) or rather high $(26 \%)$. Therefore it can be concluded that such solutions are useful in customer service.

Similarly, the ratio of respondents to chatbots on Messenger was examined. First, they were asked whether they had ever had a dialogue with a chatbot on Facebook or Messenger (a screenshot from the example of such a conversation was helpfully inserted in the questionnaire). Answers have been compiled on Figure 7.

Most of the respondents $(60 \%)$ never had conversations with chatbot on Messenger. Only nearly every third surveyed student used this type of functionality (31\%). It shows that the awareness and knowledge among students about chatbots in Messenger is generally low, which may be surprising, as respondents very often and on a large scale use this communicator. Moreover, the results of qualitative research published in the Report "Polish chatbots 2018" are confirmed, from which it appears that although respondents recruited for research $(\mathrm{N}=20)$ are active users of the Messenger communicator, "the majority of them during the study have been in contact with chatbot for the first time in their life". The authors concluded that "chatbots are still a novelty". However, from these surveys it also follows that in spite of this the respondents did not have difficulties in communicating with a chatbot and carrying out the tasks entrusted to them (business scenarios). The vast majority of users received chatbots very positively. The participants of the research declared that in the future a similar task (e.g. the purchase of a cinema ticket) would be readily performed again in this new contact channel. Also important was the conclusion that chatbots have low "entry barriers" for new users, and Internet users are already open to first contacts with chatbots ${ }^{13}$.

${ }^{13}$ Ibidem. 


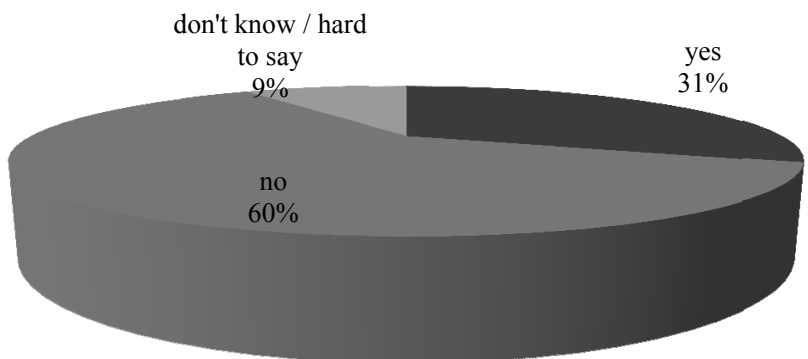

Figure 7. Conducting conversations with chatbot on Messenger by respondents $(\mathrm{N}=120)$ Source: Author's own research.

Chatbots on Messenger were also positively assessed by surveyed respondents in the context of customer service. Those who used this communication channel $(\mathrm{N}=37)$ were asked about their feelings from this conversation (Figure 8) and to what extent they managed to settle the matter thanks to this channel (Figure 9).

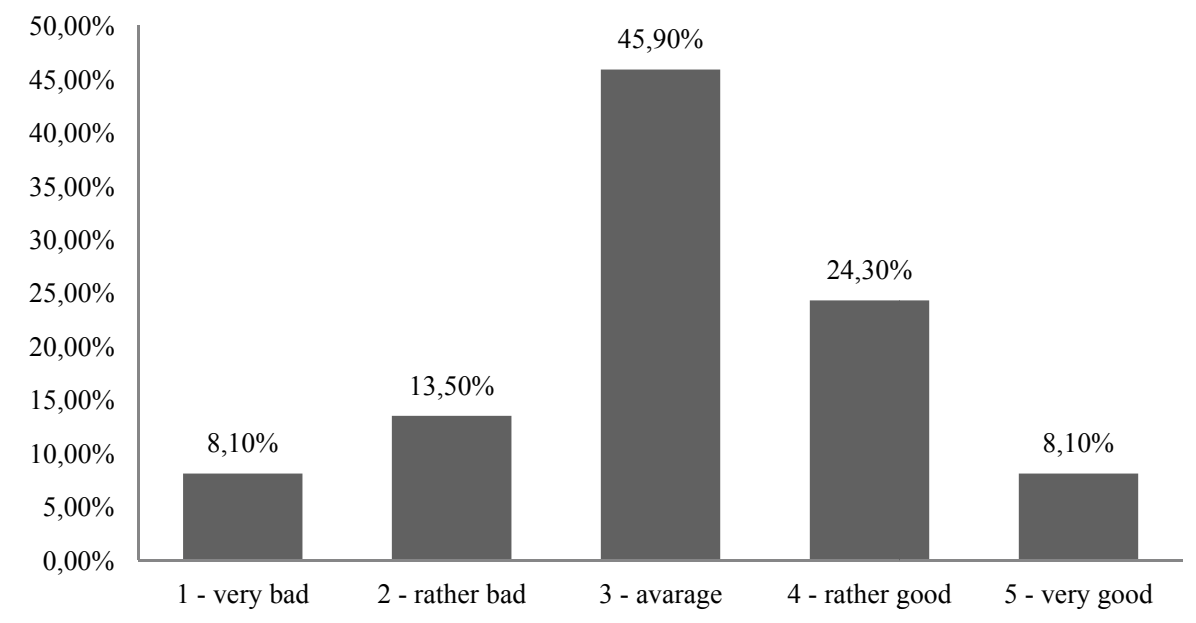

Figure 8. The feelings of the respondents in relation to conversations with chatbot on Messenger $(\mathrm{N}=37)$ Source: Author's own research.

It turns out that $46 \%$ of respondents who had conversations with chatbot on Messenger had average feelings, and $24 \%$ - rather good. Their overall impressions can be assessed as rather positive. 


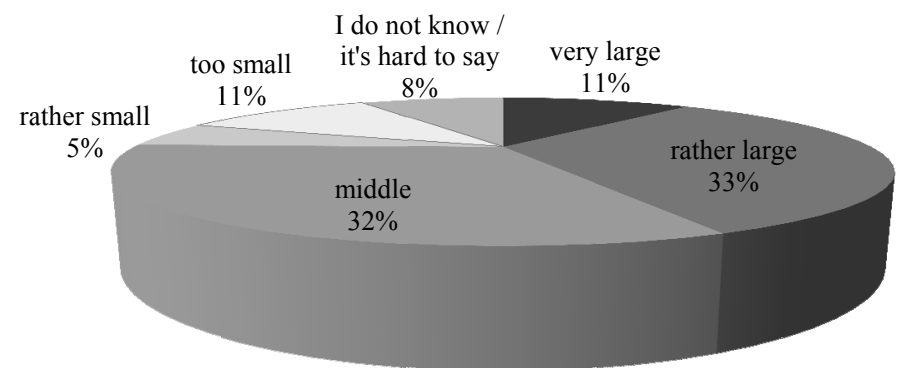

Figure 9. The extent to which respondents managed to settle the matter through conversation with the chatbot on Messenger $(\mathrm{N}=37)$

Source: Author's own research.

Every third respondent stated that thanks to chatbot on Messenger things were managed to a rather large extent (32\%) or medium (32\%). The general usefulness of Chatbots on Messenger is also assessed positively.

Based on the presented research results, H3 hypothesis can be accepted that respondents positively assess the usefulness of chatbots popping up on websites and chatbots in Messenger in customer service.

It was also important to verify the impact of chatbots on the image of a brand or company that uses them in customer service (Figure 10).

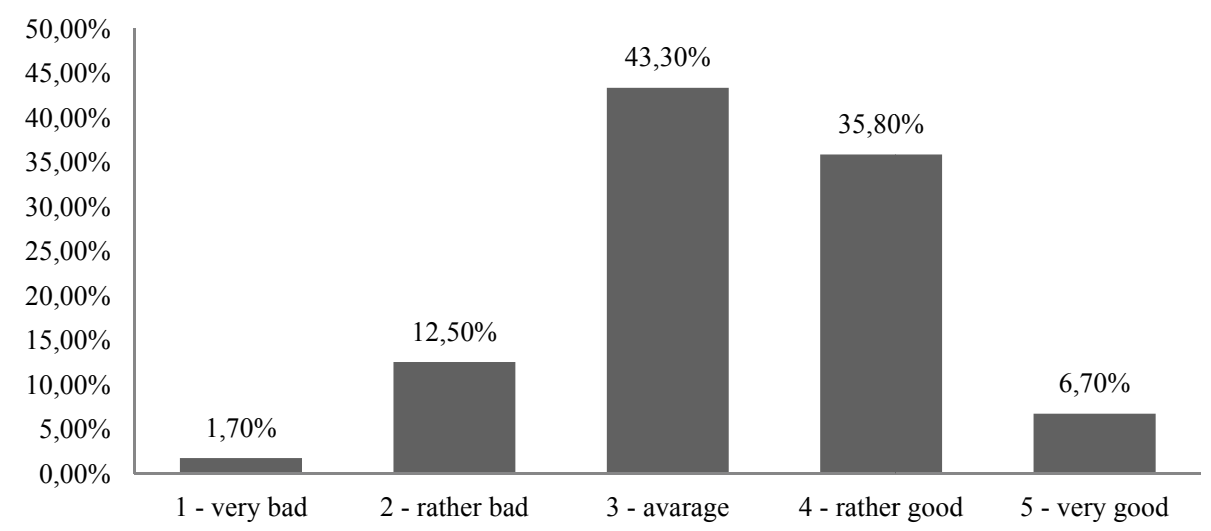

Figure 10. Respondents' assessment of the impact of using chatbots on the image of the brand / company that uses them in customer service $(\mathrm{N}=120)$

Source: Author's own research.

Almost half of respondents (43\%) assess the impact of using chatbots on the image of a brand or company that uses them in customer service, as medium, and $36 \%$ - as good. It seems that - with some caution - one can accept the H4 hypothesis that chatbots have a beneficial effect on the image of a brand or company that uses them in customer service.

Finally, the respondents were asked what they think about the use of chatbots in the area of customer service: are they helpful and sympathetic (nice), or rather irritating, and 
above all, whether they can be a good alternative to helpline and whether their development in this direction is desirable. Answers have been compiled on Figure 11.

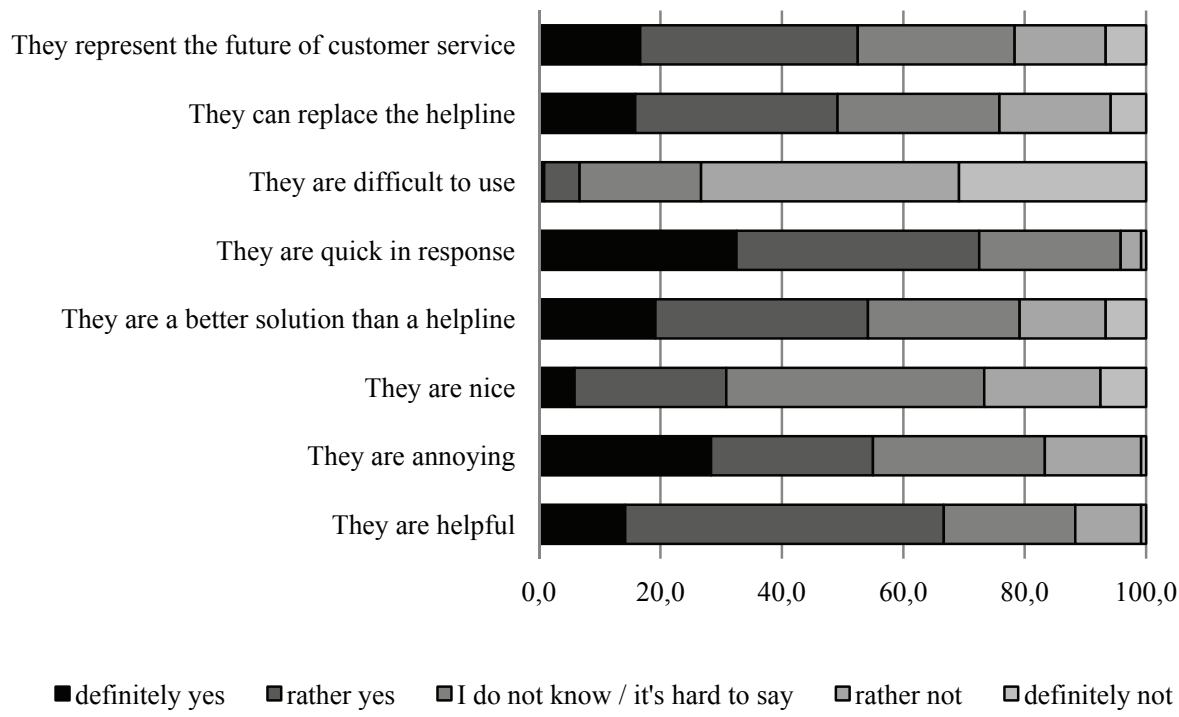

Figure 11. Respondents' assessment of using chatbots in the field of customer service $(\mathrm{N}=120)$

Source: Author's own research.

According to $53 \%$ of surveyed students, chatbots are helpful in customer service, and according to $43 \%$ - they are nice. However for many of the respondents chatbots can be annoying ( $28 \%$ - definitely yes, $27 \%$ - rather yes). Probably it is mainly due to the opinion about chatbots on websites (they can disturb users when they pop up). Respondents appreciate the fact that chatbots respond quickly ( $40 \%$ - rather yes, $33 \%$ definitely yes) and are not difficult to use ( $43 \%$ - probably not, $31 \%$ - definitely not). This confirms the previously quoted conclusions from the Report that chatbots although they are new to many users - have a "low threshold" to entry, as they are userfriendly ${ }^{14}$.

Over half of the respondents think that chatbots are a better solution than helpline (35\% - rather yes, $19 \%$ - definitely yes). They can also replace helpline (33\% - rather yes, $16 \%$ - definitely yes). Students admit that they represent the future in customer service ( $36 \%$ - rather yes, $17 \%$ - definitely yes).

The above research results allow us to accept the $\mathrm{H} 5$ hypothesis that chatbots are a good alternative to other communication channels in the field of customer service, and their development in this direction is desirable. Therefore, all hypotheses have been verified positively.

\footnotetext{
${ }^{14}$ Raport. Polskie chatboty 2018, K2 Digital Transformation, p. 72.
} 


\section{Conclusions}

The obtained research results have achieved the purpose of the research, which was the recognition of the respondents' attitude to chatbots in the context of customer service and defining the development prospects for their use in this area. Studies have shown that respondents, despite the very frequent use of various types of internet services (e.g. e-mail, Facebook, Messenger, internet shopping), in many cases cannot determine if they have ever encountered any chatbot (H1). Respondents can determine contact with a given chatbot only after being asked for it directly (H2). At the same time their attitude towards chatbots is rather positive. Rather, they positively assess the usefulness of chatbots popping up on websites (although these are sometimes irritating) and chatbots in Messenger in customer service (H3). Chatbots also have a beneficial effect on the image of the brand or company that uses them in customer service (H4). Moreover, they are a good alternative to other communication channels in the field of customer service (such as helpline), and their development in this direction is desirable (H5). Surprising in the research is that students - although they are frequent Messenger users - still have low awareness about chatbots in this communicator. They are still new to them. On the other hand, chatbots are userfriendly, which definitely supports the belief that their role in customer service should increase. It will have a good effect on the image of the brand / company. Thus, the main hypothesis was confirmed.

\section{References}

Abdul-Kader S.A., Woods J.C.: Survey on chatbot design techniques in speech conversation systems." International Journal of Advanced Computer Science and Applications 6.7 (2015).

$\mathrm{Xu}$, Anbang, et al.: A new chatbot for customer service on social media. Proceedings of the 2017 CHI Conference on Human Factors in Computing Systems. ACM, 2017.

Brandtzaeg P.B., Følstad A.: Why people use chatbots, International Conference on Internet Science. Springer, Cham, 2017.

Braun A., Chatbots in der Kundenkommunikation. Springer-Verlag, 2013.

Dale R.: The return of the chatbots, Natural Language Engineering 22.5 (2016).

Hill J., Ford W.R., Farreras I.G.: Real conversations with artificial intelligence: A comparison between human-human online conversations and human-chatbot conversations." Computers in Human Behavior 49 (2015): 245-250.

Huang J., Zhou M., Yang D.: Extracting Chatbot Knowledge from Online Discussion Forums, IJCAI. Vol. 7. 2007.

Radziwill N.M., Benton M.C.: Evaluating quality of chatbots and intelligent conversational agents. arXiv preprint arXiv:1704.04579 (2017).

Raport. Polskiechatboty 2018, K2 Digital Transformation, 2018.

Shawar B.A., Atwell E.: Chatbots: are they really useful?, Ldv forum. Vol. 22. No. 1. 2007.

Shawar A.B., Atwell E.: Using dialogue corpora to train a chatbot, Proceedings of the Corpus Linguistics 2003 conference. 2003.

Yan M. et al.: Building a chatbot with serverless computing." Proceedings of the 1st International Workshop on Mashups of Things and APIs. ACM, 2016.

https://napoleoncat.com/stats/ (28.02.2019). 
Information about author:

\section{Dr. Iwona Lupa-Wójcik}

Institute of Law, Administration and Economy

Pedagogical University of Krakow

Podchorążych 2, 30-084 Kraków, Poland

e-mail: iwona.lupa@wp.pl

ORCID: 0000-0003-1673-7077 